\title{
Unification of step bunching phenomena on vicinal surfaces
}

\author{
Pak-Wing Fok, ${ }^{1}$ Rodolfo R. Rosales, ${ }^{2}$ and Dionisios Margetis ${ }^{3}$ \\ ${ }^{1}$ Applied and Computational Mathematics, California Institute of Technology, Pasadena, California 91125, USA \\ ${ }^{2}$ Department of Mathematics, Massachusetts Institute of Technology, Cambridge, Massachusetts 02139, USA \\ ${ }^{3}$ Department of Mathematics and Institute for Physical Science and Technology, University of Maryland, \\ College Park, Maryland 20742, USA
}

(Received 5 May 2005; published 12 July 2007)

\begin{abstract}
We unify step bunching (SB) instabilities occurring under various conditions on crystal surfaces below roughening. We show that when attachment-detachment of atoms at step edges is the rate-limiting process, the $\mathrm{SB}$ of interacting, concentric circular steps is equivalent to the commonly observed SB of interacting straight steps under deposition, desorption, or drift. We derive a continuum Lagrangian partial differential equation, which is used to study the onset of instabilities for circular steps. These findings place on a common ground SB instabilities from numerical simulations for circular steps and experimental observations of straight steps.
\end{abstract}

DOI: 10.1103/PhysRevB.76.033408

PACS number(s): 81.10.Aj, 81.07.-b

Recent advances in the fabrication of small devices such as quantum dots have motivated theoretical research into the fundamental properties of surfaces at the nanoscale. Below the roughening transition temperature, ${ }^{1}$ the evolution of crystal surfaces is governed by the motion of steps. ${ }^{2}$ This motion is important in understanding phenomena such as pattern formation $^{3}$ and the self-assembly of nanostructures. ${ }^{4}$

One of the most commonly studied surface phenomena is step bunching (SB), where steps cluster together tightly into widely separated bunches. ${ }^{5}$ This instability has been observed experimentally on many different systems, e.g., see Refs. 6-8 Most theoretical studies of SB have focused on the idealized situation with straight steps. ${ }^{3,9,10}$ However, as surface features become smaller, the step curvature should play an increasingly important role. ${ }^{11}$ Therefore, a realistic model of step bunching must include both the effects of step curvature and step interactions. ${ }^{1}$

Experimentally, the most common way to induce SB is to heat the surface using a direct current. ${ }^{12}$ The resulting instability in straight steps has been understood on the basis of an asymmetry in the adatom density caused by a preferential drift. ${ }^{13}$ However, the equivalent phenomenon in circular steps has received much less attention. Experiments for circular steps are not uncommon ${ }^{14,15}$ and yet, very few theories currently exist that predict the onset of SB in circular steps (see Ref. 16, however, for a quasi-steady-state analysis).

In this Brief Report, we discuss how curvature differences from one step to another can also induce a drift and, thus, give rise to SB. The main result is the derivation of a reduced partial differential equation (PDE), Eq. (12) below, which captures this effect and is able to predict the onset of SB instabilities for relaxing circular steps. We therefore unify SB phenomena in straight and circular steps by showing that they have a common physical and mathematical basis. In particular, our approach demonstrates that the effect of step line tension on SB is equivalent to that of a drift or of desorption and/or material deposition in the presence of a difference in the kinetic rates at step edges. This equivalence is shown by treating the step index as a continuum variable. ${ }^{17}$ In the resulting PDEs for the step positions, the step line tension and the physical effects described above all contribute to a destabilizing, "backward" diffusion term. Our ap- proach, therefore, differs from Ref. 18, which categorized a broad range of SB instabilities according to their universality class.

We first review briefly SB instabilities of straight steps ${ }^{19-21}$; see Table I. We stress that all the results in this table are already well established. Our intentions here are (i) to derive continuum equations for straight-step equations in the Lagrangian framework so that the analogy between an externally induced drift in straight steps and curvatureinduced drift in circular steps is made clear; and (ii) study the step bunching of circular steps ${ }^{22}$ on the basis of the PDE in Lagrangian coordinates.

Under the quasi-steady-state approximation, ${ }^{13}$ the onedimensional adatom density, $c_{i}$, on the $i$ th terrace satisfies

$$
D \frac{\partial^{2} c_{i}}{\partial x^{2}}-v_{0} \frac{\partial c_{i}}{\partial x}-\frac{c_{i}}{\tau}+R=0
$$

where $D$ is the terrace diffusivity, $v_{0}$ is the down-step drift velocity, $R$ is the deposition flux, and $\tau$ is the desorption time. The adatom current is $J_{i}(x)=-D \frac{\partial c_{i}}{\partial x}+v_{0} c_{i}$. We then solve for $c_{i}(x)$ by imposing boundary conditions for adatom attachment-detachment at step edges:

$$
-\left.J_{i}\right|_{x_{i}}=k_{+}\left(\left.c_{i}\right|_{x_{i}}-C_{i}^{\mathrm{eq}}\right)
$$

TABLE I. A summary of some known conditions under which SB occurs in numerical simulations; ES, Ehrlich-Schwoebel barrier. A cross (check) indicates absence (presence) of the corresponding physical effect. The conditions most relevant to experiments (Ref. 12) are those in (Refs. 19 and 21).

\begin{tabular}{cccccc}
\hline \hline ES & Curvature & Drift & Desorption & Deposition & Reference \\
\hline$\times$ & $\times$ & $\sqrt{ }$ & $\sqrt{ }$ & $\sqrt{ }$ & 19 \\
$\downarrow$ & $\times$ & $\times$ & $\times$ & $\sqrt{ }$ & 20 \\
$\times$ & $\times$ & $\sqrt{ }$ & $\sqrt{ }$ & $\times$ & 21 \\
$\times$ & $\downarrow$ & $\times$ & $\times$ & $\times$ & 22 \\
\hline \hline
\end{tabular}




$$
\left.J_{i}\right|_{x_{i+1}}=k_{-}\left(\left.c_{i}\right|_{x_{i+1}}-C_{i+1}^{\mathrm{eq}}\right) .
$$

Here, $k_{+}$and $k_{-}$are rate coefficients for attachmentdetachment from the lower and upper terraces, and $C_{i}^{\text {eq }}$ is the equilibrium adatom density at the $i$ th step, to be given below. If $k_{+}>k_{-}$, then we have a "positive" Ehrlich-Schwoebel (ES) barrier, ${ }^{23,24}$ whereas $k_{+}<k_{-}$results in a "negative" ES barrier. The propensity of steps to accept adatoms is described by the step chemical potential, $\mu_{i}$. We write $C_{i}^{\mathrm{eq}}=c_{0} e^{\mu_{i} / k_{B} T}$ $\approx c_{0}\left(1+\frac{\mu_{i}}{k_{B} T}\right)$, where $k_{B} T$ is the Boltzmann energy, $c_{0}$ is the density at an isolated step, and ${ }^{1}$

$$
\frac{\mu_{i}}{k_{B} T}=g\left[\left(\frac{L}{x_{i+1}-x_{i}}\right)^{3}-\left(\frac{L}{x_{i}-x_{i-1}}\right)^{3}\right] \text {. }
$$

In Eq. (4), $L$ is the typical terrace width and $g$ is the (dimensionless) strength of step interactions. Once the adatom density $c_{i}(x)$ is known, the step velocity is

$$
\frac{d x_{i}}{d t}=\frac{\Omega}{a}\left[J_{i-1}\left(x_{i}\right)-J_{i}\left(x_{i}\right)\right],
$$

where $\Omega$ is the atomic volume and $a$ is the step height. Equation (5) along with Eqs. (1)-(4) yields a system of coupled equations for the step positions.

Next, we express the continuum limit of Eq. (5) through Lagrangian coordinates. We thus replace the discrete $x_{i}(t)$ by a smooth function $x(s, t)$. Specifically, $x_{i}(t)=x(s, t)$, where $s$ $=i \delta$ and $\delta \ll 1$; hence, $s$ becomes the dimensionless, continuous version of the discrete index $i$. Then, in Eq. (5), terms such as $x_{i+1}(t)$ are replaced by their Taylor expansions, e.g., $x(s+\delta, t)=x(s, t)+\delta x_{s}(s, t)+\ldots$, where the subscript $s$ denotes partial derivative. This continuum approximation is valid in regions where significant changes in $x_{i+1}-x_{i}$ happen over a large, $O\left(\delta^{-1}\right)$, number of steps. In the case of step bunching, the approximation is valid inside each bunch, but breaks down across terraces that connect individual bunches, as discussed below. The merits of using a Lagrangian continuum approach are as follows: (i) large single terraces, which invalidate the continuum, conveniently correspond to very thin regions in the Lagrangian coordinate $s$; and (ii) the derivation of (leading-order) continuum equations can be carried out via Taylor expansions and, hence, be easily automated.

We assume that terrace diffusion is much faster than attachment-detachment at step edges; that is, the step kinetics is attachment-detachment limited (ADL). Therefore, the ratio of the typical terrace width, $L$, to the diffusion length $\sqrt{D \tau}$ is small; we take $\delta$ to be precisely this ratio. The resulting Lagrangian equations of motion are nonlinear. If we linearize around a solution corresponding to equally spaced steps, then we obtain ${ }^{21}$

$$
u_{t}=A_{1} u_{s}-A_{2} u_{s s}+A_{3} u_{s s s}-A_{4} u_{s s s s} .
$$

Here, $u(s, t) \equiv x(s+\delta, t)-x(s, t)$ is the terrace width, and the coefficients $A_{i}$ are of leading order in $\delta$ and depend on the physical effects included. A nonlinear equation to describe step bunching has been derived in Ref. 25. In obtaining Eq. (6), we have chosen a time scale for the step motion equal to $\frac{a L}{\Omega c_{0} \delta^{2}} \sqrt{\frac{\tau}{D}}$. The stability of the terrace widths, as predicted by a linear analysis, depends only on the signs of the coefficients $A_{2}$ and $A_{4}$; neither $A_{1}$ nor $A_{3}$ affects it. More precisely, the amplitude of modes with wave number $k$ grows as $e^{\left(A_{2} k^{2}-A_{4} k^{4}\right) t}$. The coefficients $A_{2}$ and $A_{4}$ are given by

$$
A_{2}=-\delta\left[3 g+\frac{1}{2}\left(\frac{R \tau}{c_{0}}-1\right)\left(\frac{k_{+}-k_{-}}{k_{+}+k_{-}}\right)-\frac{v_{0} \tau}{D}\left(\frac{k_{+} k_{-}}{k_{+}+k_{-}}\right)\right]
$$

$$
A_{4}=\delta^{2} g \sqrt{\frac{\tau}{D}}\left[\frac{3 k_{-} k_{+}}{k_{+}+k_{-}}\right]
$$

Equation (6) along with Eqs. (7) and (8) confirms the first three results in Table I. For future reference, we note that a positive drift $\left(v_{0}>0\right)$ contributes as a destabilizing backward diffusion term in Eq. (6).

We now turn our attention to the main focus of this Brief Report: the axisymmetric case, when the structure consists of a large number of concentric, circular descending steps. ${ }^{14,26}$ The relaxation of these structures is simulated in Ref. 22; see Table I. In this case, SB occurs even in the absence of deposition, desorption, drift, and ES barriers, because of the competition between step curvature and interactions. To our knowledge, this phenomenon has not been observed experimentally. However, using the continuum Lagrangian approach, we show that this curvature-driven SB can be explained on the basis of an asymmetry, in the same fashion as the experimentally observable instabilities for straight steps. $^{12}$

The derivation of the equations of motion for circular steps is similar to that of straight steps. The starting point is Eq. (5) with $x_{i}$ replaced by $r_{i}$, the radius of the $i$ th step, and $J_{i}(r)=\alpha_{i} / r$, where $r$ is the polar distance and $\alpha_{i}$ depends on $r_{i}, r_{i \pm 1}$, and $r_{i+2} \cdot{ }^{27}$ Instead of Eq. (4), we use 27,28

$$
\begin{gathered}
\mu_{i}=\frac{\Omega g_{1}}{r_{i}}+\frac{\Omega}{2 \pi a r_{i}} \frac{\partial\left[V\left(r_{i}, r_{i+1}\right)+V\left(r_{i-1}, r_{i}\right)\right]}{\partial r_{i}}, \\
V\left(r_{i}, r_{i+1}\right)=\frac{4 \pi a^{3} g_{3}}{3} \frac{r_{i} r_{i+1}}{\left(r_{i}+r_{i+1}\right)\left(r_{i+1}-r_{i}\right)^{2}} .
\end{gathered}
$$

In the first term of Eq. (9), $g_{1} a$ is the step line tension (energy/length), which has no counterpart in Eq. (4); this term causes the first step $\left(r=r_{1}\right)$ to shrink. The second term in Eq. (9) corresponds to step interactions. In Eq. (10), $g_{3}$ is the step interaction free energy; we define $\widetilde{g} \equiv \frac{2 g_{3}}{3 g_{1}}\left(\frac{a}{L}\right)^{2}$ as the relative strength of interactions to line tension, where $L$ is the typical terrace width.

To take the continuum Lagrangian limit of the step equations, we first define the dimensionless step radii $\rho_{i}(t)$ $=r_{i}(t) / L$ and expand $\rho_{i+1}(t)=\rho(s+\delta, t)=\rho(s, t)+\delta \rho_{s}+\ldots$. The resulting $\operatorname{PDE}$ for $\rho(s, t)$ is 


$$
\rho_{t}=-\frac{\delta^{2} \gamma}{\rho}\left(\frac{1}{2 q+\rho_{s}}\left\{\frac{\rho_{s}}{\rho}+\frac{3 \widetilde{g}}{\delta^{2}}\left[\frac{1}{2} \frac{1}{\rho \rho_{s}}+\left(\frac{\rho \rho_{s}}{\rho_{s}^{4}}\right)_{s}\right]\right\}\right)_{s},
$$

where $q=D /(k L)$ and $\gamma=\left(\frac{\Omega g_{1}}{k_{B} T a}\right)\left(\frac{D}{L U}\right)\left(\frac{\Omega c_{0}}{a}\right)$ are dimensionless material parameters, $k$ is the attachment-detachment rate, and $U$ is a typical step velocity. Equation (11) in Eulerian coordinates was given in Ref. 27. Note that, when $\widetilde{g} \rightarrow 0$ and $q$ $>O\left(\rho_{s}\right)$, the right side of Eq. (11) reduces to a destabilizing, backward diffusionlike term; this term causes SB in agreement with Ref. 22. The stability analysis of Eq. (11) is complicated by the fact that equally spaced steps do not form a solution.

Hence, while retaining the main effects of curvature and interactions, we make four simplifications. The resulting simplified PDE-see Eq. (12) below-contains the leading nonlinearity responsible for the onset of wide terraces separating bunches. First, we assume that the kinetics is ADL, $\rho_{s} \ll 2 q$. Second, we take $\nu \equiv 3 \widetilde{g} / \delta^{2} \ll 1$. Third, we study the stability of a set of consecutive steps that have approximately the same radius, $\rho_{0}$. Fourth, we focus on the transition regime where the step line tension balances step interactions. Thus, we seek a solution to Eq. (11) in the form $\rho=\rho_{0}$ $+\nu^{1 / 4} \rho_{1}(s, t)+O\left(\nu^{1 / 2}\right)$, where $\rho_{0}=O(1)$ is a constant and $\rho_{1}$ $=O(1)$. With $u=\partial \rho_{1} / \partial s$, we have

$$
u_{t}=\left(\frac{\delta^{2} \gamma}{2 q}\right)\left[-\frac{u_{s s}}{\rho_{0}^{2}}-\left(\frac{u_{s}}{u^{4}}\right)_{s s s}+O\left(\nu^{1 / 4}\right)\right] .
$$

Next, we discuss Eq. (12). The term $-u_{s s} / \rho_{0}^{2}$ represents the step line tension and corresponds to the term $-A_{2} u_{s s}$ in Eq. (6). The term $-\left(u_{s} / u^{4}\right)_{s s s}$ expresses the step interactions, is stabilizing, and corresponds to $-A_{4} u_{s s s s}$. Hence, by comparison with the straight-step case, we associate step curvature with the other effects commonly known to cause SB. More precisely, changes in the step curvature cause variations with $i$ in the step chemical potential $\mu_{i}$ through Eq. (9). These variations always induce nonzero currents and, thus, cause an asymmetry in the adatom density, amounting to a drift. The successive time-monotone collapse of the innermost step means that the relaxation is effectively driven by the expanding facet ${ }^{14}$ which ultimately acts as the current source for all other steps.

Figure 1 shows the result of integrating Eq. (12) numerically; note the development of a SB instability as a growing spike in $u$, i.e., an expanding terrace. Linearizing Eq. (12) around $u=$ constant. yields a special case of Eq. (6); hence, we have unified all the step bunching phenomena in Table I. However, keeping the nonlinearity in Eq. (12) is essential for the localized nature of the instability shown in Fig. 1. As the spike forms, the value of $u$ is lowered nearby ( $u$ should be conserved), and this amplifies the (nonlinear) stabilizing term. We expect that a similar nonlinear effect plays an important role in the case with straight steps.

To further validate the continuum Lagrangian approach, we use Eq. (11) with ADL kinetics to predict two scaling laws for sufficiently small $\widetilde{g}$. From step simulations, for example, Ref. 26 or Fig. 5f in Ref. 22, we observe that steps within bunches move very slowly. Thus, the adatom current

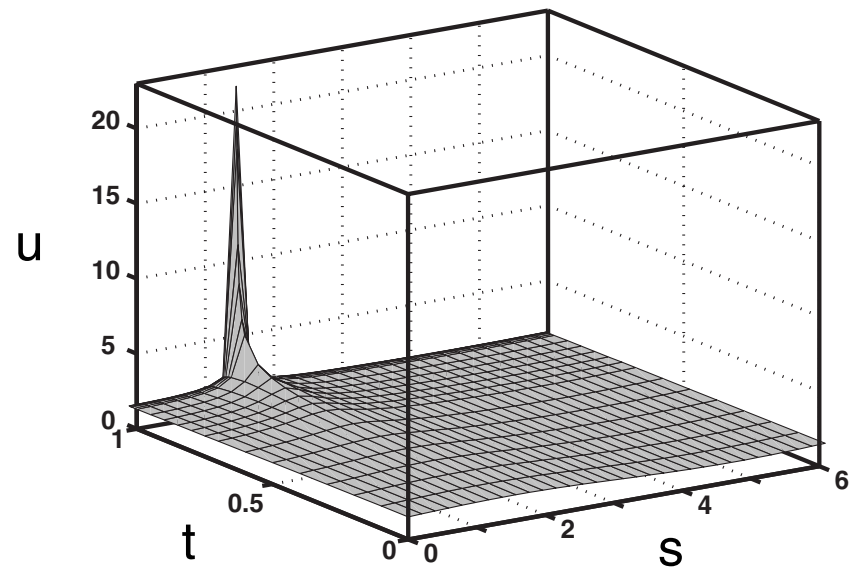

FIG. 1. Numerical solution of Eq. (12) with periodic boundary conditions in $s$. By using scaled dependent and independent variables, one can take $\rho_{0}=1$ and $\frac{\delta^{2} \gamma}{2 q}=1$ without loss of generality. The initial condition is $u(s, 0)=0.8+0.6 \sin s$.

inside the bunch is nearly a constant, $K .{ }^{29}$ Because bunches behave like individual steps, ${ }^{22}$ the radius of the first bunch decreases due to an effective line tension, independently of $\widetilde{g}$ as $\widetilde{g} \rightarrow 0$; therefore, the adatom current must also be independent of $\tilde{g}$ to leading order. We infer that $K=O(1)$ when $\tilde{g}$ $\ll 1$. Hence we set the term in the curly brackets of Eq. (11) equal to a constant proportional to $K$. By approximately solving the resulting differential equation for ADL kinetics, we obtain $\rho_{s}=O\left(\tilde{g}^{1 / 3} N^{-2 / 3}\right)$, where $N$ is the number of steps in the bunch. Thus, we obtain two scaling predictions. First, the bunch width scales as $\widetilde{g}^{1 / 3}$, in agreement with simulation data; see Fig. 2. The same scaling is given in Refs. 10 and 21 , but for straight steps. Second, for fixed $\widetilde{g}$, the typical terrace width inside a bunch scales as $N^{-2 / 3}, 30$ in agreement with the straight-step cases. ${ }^{10,21,29}$

So far, we have only considered SB with ADL kinetics. Next, we study the more physically relevant case with "mixed" kinetics, which incorporates the joint effect of attachment-detachment and terrace diffusion; the latter acts as an additional stabilizing influence on the step motion. As in the ADL case, SB occurs if step line tension dominates,

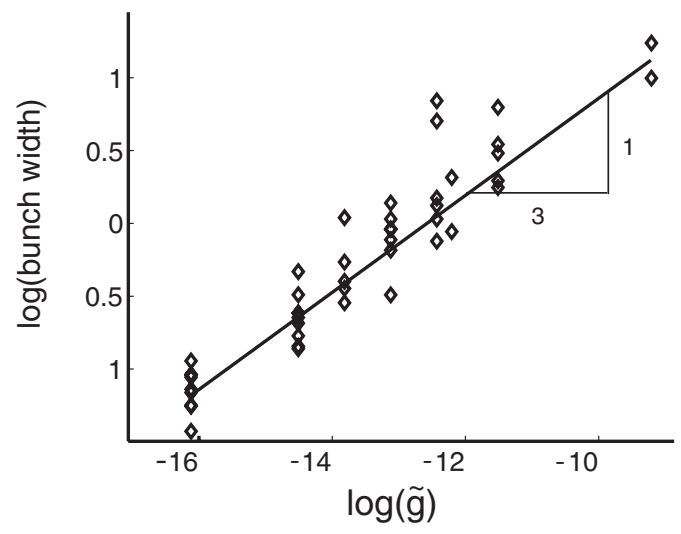

FIG. 2. Log-log plot of step bunch width vs step interaction strength $\widetilde{g}$ under ADL kinetics. Symbols represent simulation data at a fixed time $t$, and the solid line is our scaling prediction. 
but now this instability can be suppressed due to terrace diffusion. Diffusion becomes more important if either the terrace diffusivity decreases or the terrace width increases sufficiently: both of these conditions are expressed by the local condition $q / \rho_{s} \ll 1$. Figure 3(a) shows the evolution of the terrace widths over time, obtained by integrating the discrete circular-step equations numerically with $q=1$. The initial condition used is $\rho_{n}=n+\sin (0.1 n)$, which amounts to $\partial \rho / \partial s$ $=1+0.1 \cos (0.1 s)$. Hence, $q / \rho_{s} \approx 1$ initially and we see that SB forms even when diffusion and attachment-detachment are comparable. The development of a spike in the terrace width is similar to the behavior in ADL kinetics; compare Fig. 3(a) with Fig. 1.

The transition to diffusion-limited kinetics can be seen through Eq. (11). In the limit $q / \rho_{s} \rightarrow 0$, the line tension term reduces from $\frac{1}{\rho} \frac{\partial}{\partial s}\left(\frac{\rho_{s} / \rho}{2 q+\rho_{s}}\right)$ to $\frac{1}{\rho} \frac{\partial}{\partial s}\left(\frac{1}{\rho}\right)$, the destabilizing effect of backward diffusion is removed, and, in fact, step bunches decay over time due to step repulsions. The initial condition used in Fig. 3(b) is identical to that in Fig. 3(a), but now $q$ $=0.1$. In contrast to Fig. 3(a), the bunches now do not steepen and, instead, small perturbations to a constant $\rho_{s}$ profile decay exponentially with a rate constant $\propto \widetilde{g}$ in the limit $q / \rho_{s} \rightarrow 0 .{ }^{26}$ The theoretical prediction of diffusion-limited SB decay made by Eq. (11) along with the simulation result in Fig. 3(b) is the circular-step analog of the bunch decay observed experimentally in Ref. 6.

In conclusion, we have unified various conditions under which SB occurs. In particular, SB arising from drift and curvature effects can be explained using the same underlying principles. We believe that the continuum Lagrangian approach used here opens up promising research directions. For example, although we unified the SB instabilities in Table I, the instabilities arising from long-range ${ }^{8}$ and attractive ${ }^{9}$ in-
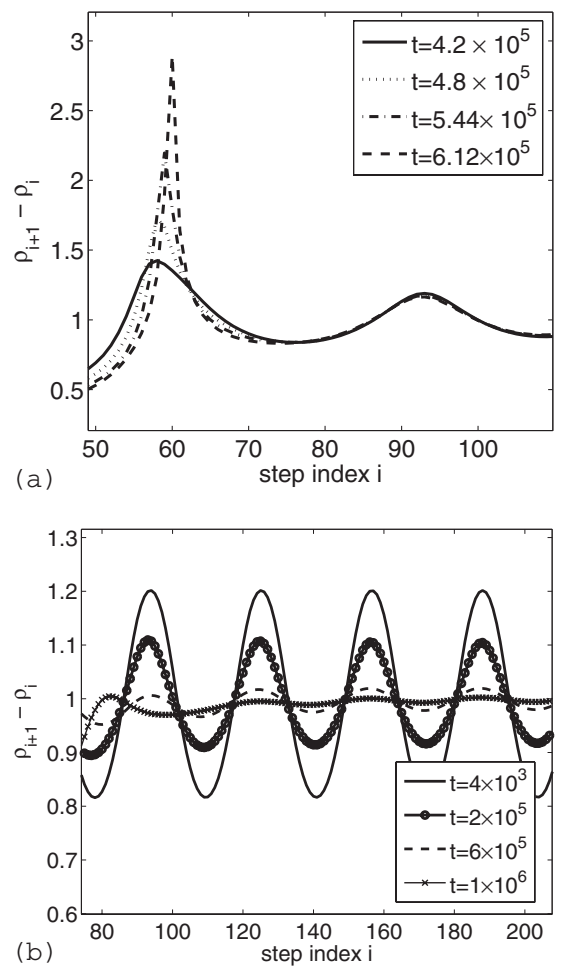

FIG. 3. SB formation and decay for (a) $q / \rho_{s} \approx 1$ and (b) $q / \rho_{s}$ $\approx 0.1$. In both cases, $\widetilde{g}=10^{-3}$ and an initial condition of $\rho_{n}=n$ $+\sin (0.1 n)$ was used.

teractions were not discussed. Also, the derivation of conditions that connect Lagrangian solutions inside adjacent bunches across wide terraces is work in progress.
${ }^{1}$ H.-C. Jeong and E. D. Williams, Surf. Sci. Rep. 34, 171 (1999).

${ }^{2}$ W. K. Burton, N. Cabrera, and F. C. Frank, Philos. Trans. R. Soc. London, Ser. A 243, 299 (1951).

${ }^{3}$ D. Kandel and J. D. Weeks, Phys. Rev. Lett. 69, 3758 (1992).

${ }^{4}$ M. Kitamura et al., Appl. Phys. Lett. 66, 3663 (1995).

${ }^{5}$ M. Uwaha and M. Sato, Surf. Rev. Lett. 5, 841 (1998).

${ }^{6}$ E. S. Fu, M. D. Johnson, D. J. Liu, J. D. weeks, and E. D. Williams, Phys. Rev. Lett. 77, 1091 (1996).

${ }^{7}$ A. A. Darhuber et al., Appl. Phys. Lett. 73, 1535 (1998).

${ }^{8}$ W. Hong, H. Lee, M. Yoon, H. Christen, D. Lowndes, Z. Suo, and Z. Zhang, Phys. Rev. Lett. 95, 095501 (2005).

${ }^{9}$ F. Liu, J. Tersoff, and M. G. Lagally, Phys. Rev. Lett. 80, 1268 (1998).

${ }^{10}$ M. Sato and M. Uwaha, Surf. Sci. 442, 318 (1999).

${ }^{11}$ M. Degawa, F. Szalma, and E. D. Williams, Surf. Sci. 583, 126 (2005).

${ }^{12}$ A. Latyshev et al., Surf. Sci. 213, 157 (1989).

${ }^{13}$ S. Stoyanov, Jpn. J. Appl. Phys., Part 1 30, 1 (1991).

${ }^{14}$ K. Thürmer, J. E. Reutt-Robey, E. D. Williams, M. Uwaha, A. Emundts, and H. P. Bonzel, Phys. Rev. Lett. 87, 186102 (2001).

${ }^{15}$ Y. Homma, H. Hibino, T. Ogino, and N. Aizawa, Phys. Rev. B 58, 13146 (1998).
${ }^{16}$ M. Uwaha and K. Watanabe, J. Phys. Soc. Jpn. 69, 497 (2000).

${ }^{17}$ M. Ozdemir and A. Zangwill, Phys. Rev. B 42, 5013 (1990).

${ }^{18}$ A. Pimpinelli, V. Tonchev, A. Videcoq, and M. Vladimirova, Phys. Rev. Lett. 88, 206103 (2002).

${ }^{19}$ D. J. Liu and J. D. Weeks, Phys. Rev. B 57, 14891 (1998).

${ }^{20}$ M. Sato and M. Uwaha, Surf. Sci. 493, 494 (2001).

${ }^{21}$ S. Stoyanov and V. Tonchev, Phys. Rev. B 58, 1590 (1998).

${ }^{22}$ N. Israeli and D. Kandel, Phys. Rev. B 60, 5946 (1999).

${ }^{23}$ G. Ehrlich and F. G. Hudda, J. Chem. Phys. 44, 1039 (1966).

${ }^{24}$ R. L. Schwoebel and E. J. Shipsey, J. Appl. Phys. 37, 3682 (1966).

${ }^{25}$ C. Misbah and O. Pierre-Louis, Phys. Rev. E 53, R4318 (1996).

${ }^{26}$ P.-W. Fok, Ph. D. thesis, Massachusetts Institute of Technology, 2006.

${ }^{27}$ D. Margetis, M. J. Aziz, and H. A. Stone, Phys. Rev. B 71, 165432 (2005).

${ }^{28}$ S. Tanaka, N. C. Bartelt, C. C. Umbach, R. M. Tromp, and J. M. Blakeley, Phys. Rev. Lett. 78, 3342 (1997).

${ }^{29}$ J. Krug, V. Tonchev, S. Stoyanov, and A. Pimpinelli, Phys. Rev. B 71, 045412 (2005).

${ }^{30}$ This scaling has not yet been validated by our simulations for circular steps. 